\title{
The Reduction of Jet Noise with Spherical Reflector and Evaluation of its Performance Compared to a Flat Reflector
}

\author{
Kunisato Seto ${ }^{\dagger}$, Md. Tawhidul Islam Khan ${ }^{\dagger}$ and Kenbu Teramoto \\ Department of Mechanical Engineering, Saga University, 1 Honjo-machi, Saga 840-8502, Japan
}

(Received 19 October 2004; revised 12 April 2006; accepted 1 November 2006)

\begin{abstract}
A method of jet screech suppression with a spherical reflector has been proposed. The reflector was placed at the nozzle exit. Accurate placement of the reflector controlled the location of image sound source and reduced the sound pressure at the nozzle exit. The muted sound did not excite the unstable disturbance at the nozzle exit and the loop of the feedback mechanism disappeared, thus the generation of jet screech was cancelled. It was indicated that the new technique suppressed not only the screech tones, but also shielded the upstream radiation of the broadband noise components. Hence, the proposed spherical reflector technique protects the upstream noise propagation, and therefore protects the upstream structures of the nozzle exit from the acoustic fatigue. Optical investigations were carried out by using the Schlieren apparatus along with a high-speed video camera and it was observed that the jet was modified and became stable by the attachment of the spherical reflector at the nozzle exit. The performance of noise reduction with a flat reflector was also compared with the proposed technique. It was found that the method of noise suppression of a supersonic jet with the technique of the spherical reflector was more effective than that of the flat reflector.
\end{abstract}

${ }^{\dagger}$ Member of the International Institute of Acoustics and Vibration (IIAV)

\section{INTRODUCTION}

An underexpanded supersonic jet radiates jet mixing noise, broadband shock-associated noise, as well as very loud discrete screech tones under certain conditions. In this jet, a quasi-periodic shock cell structure develops in the jet plume because of the pressure mismatch at the nozzle exit. The interaction of the shock cells pattern with the large-scale structure in the shear layer causes the radiation of two additional noise components from the jet: screech tones and broadband shock-associated noise. ${ }^{1,2}$ The jet screech is of particular interest not only because of the general noise-reduction but also the acoustical fatigue of the structure. Many experimental and theoretical investigations of jet noise have been carried out for a comprehensive discussion by Panda et al. ${ }^{3,4}$ Mixing noise is directly associated with large-scale structures, or instability waves, in the jet shear layer; whereas, broadband shock-associated noise and screech tones are associated with the interaction of these waves with the shock-cell structure in the jet core. The screech tones arise because a part of the acoustic waves generated by the wave shock-cell interaction propagates upstream and regenerates the instability waves at, or in the vicinity of, the nozzle lip. This feedback loop leading to distinct screech tones is sensitive to small changes in the system conditions, and its explanation is to date mostly based on experimental observations. ${ }^{2}$

The overall, self-sustaining, feedback loop that controls the screech production mechanism was explained by Powell. ${ }^{5,6}$ Since then there had been many visualisation and experimental studies to validate the mechanism. ${ }^{7-9}$ According to the feedback loop, instabilities, created in a thin shear layer and receptive to external excitation, have the ability to propagate downstream. The created instability wave is able to pick up energy from the flow and to grow rapidly in amplitude as it propagates through the jet quasi-periodic shock cells. After passing three or four shock cells, the wave amplitude is generally sufficient for unsteady interaction to occur between the wave and the shock cell structure. A detailed observation of the feedback loop, the sound generation process and the receptivity issue was done by Panda. ${ }^{4} \mathrm{He}$ found that the feedback loop apparently chose the new length scale represented by the standing wavelength instead of the average shock spacing in selecting the screech frequency. Still some parts of screech phenomenon remain unsolved, such as where the effective sound sources exist and how the shocks respond to a periodic train of disturbances.

The characteristic of screech tone is very much unsteady. This discrete tone can be heard from overexpanded and underexpanded supersonic jets. When a jet screech is present, the sound level associated with the screech tone dominates the jet noise components in the upstream direction. Seiner, Manning, and Ponton ${ }^{\mathbf{1 0}}$ studied twin-jet screech resonance with nozzle geometries found in military aircraft and measured very high dynamic loads capable of causing tailplane structural failure. An earlier study Hay and Rose ${ }^{11}$ also made similar observations. The noise level of jet engines, particularly during the takeoff and during the climb, is often a concern for the people living near to the airport. It is well known that the intensity of screech tone decreases with the increase of the jet temperature when the jet Mach number is low. ${ }^{12}$ However, the intended high-speed civil transport program calls the attention again to the noise reduction. ${ }^{13}$ One of the most frustrating aspects of the research of supersonic jet noise is the inability to predict the intensity of screech tones. The difficulties appear to lie both in the extreme sensitivity of the screech tones to the surrounding environment and in the inherent need to deal with the nonlinearities of the feed- 